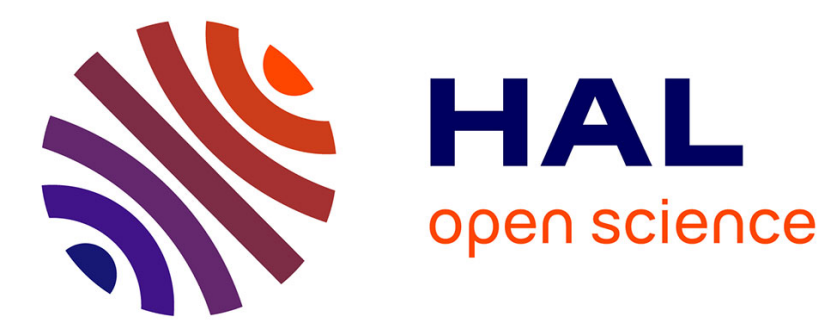

\title{
Molecular Interactions of $\beta$-Cyclodextrins with Monolayers Containing Adamantane and Anthraquinone Guest Groups
}

\author{
Renata Bilewicz, Olga Swiech, Kazimierz Chmurski
}

\section{- To cite this version:}

Renata Bilewicz, Olga Swiech, Kazimierz Chmurski. Molecular Interactions of $\beta$-Cyclodextrins with Monolayers Containing Adamantane and Anthraquinone Guest Groups. Supramolecular Chemistry, 2010, 22 (07-08), pp.461-466. 10.1080/10610278.2010.486138 . hal-00602593

\section{HAL Id: hal-00602593 https://hal.science/hal-00602593}

Submitted on 23 Jun 2011

HAL is a multi-disciplinary open access archive for the deposit and dissemination of scientific research documents, whether they are published or not. The documents may come from teaching and research institutions in France or abroad, or from public or private research centers.
L'archive ouverte pluridisciplinaire $\mathbf{H A L}$, est destinée au dépôt et à la diffusion de documents scientifiques de niveau recherche, publiés ou non, émanant des établissements d'enseignement et de recherche français ou étrangers, des laboratoires publics ou privés. 


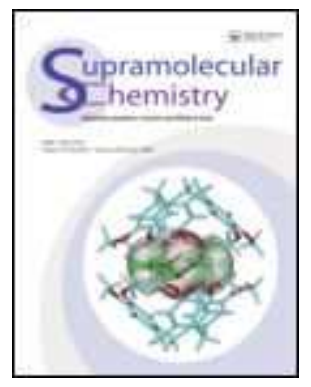

Molecular Interactions of $\beta$-Cyclodextrins with Monolayers Containing Adamantane and Anthraquinone Guest Groups

\begin{tabular}{|r|l|}
\hline Journal: & Supramolecular Chemistry \\
\hline Manuscript ID: & GSCH-2010-0021.R1 \\
\hline Manuscript Type: & Special Issue Paper \\
\hline Date Submitted by the & 09-Apr-2010 \\
\hline Complete List of Authors: & $\begin{array}{l}\text { Bilewicz, Renata; University of Warsaw, Chemistry } \\
\text { Swiech, Olga; University of Warsaw } \\
\text { Chmurski, Kazimierz; University of Warsaw }\end{array}$ \\
\hline Keywords: & $\begin{array}{l}\text { cyclodextrin, anthraquinone, adamantane, self assembled } \\
\text { monolayer, association constant }\end{array}$ \\
\hline
\end{tabular}

\section{S) ScholaroNE \\ Manuscript Central}




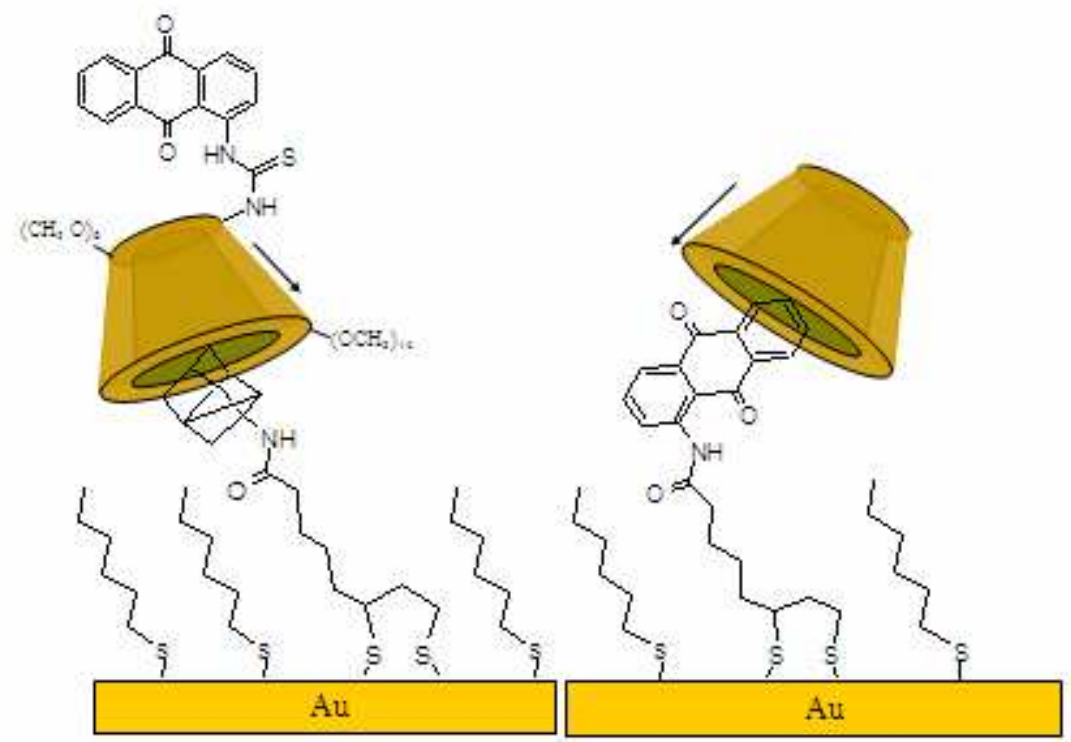




\title{
Molecular Interactions of $\beta$-Cyclodextrins with Monolayers Containing Adamantane and Anthraquinone Guest Groups
}

\author{
Olga Swiech, Kazimierz Chmurski, Renata Bilewicz* \\ Department of Chemistry, University of Warsaw, Pasteura 1, 02-093 Warsaw, Poland \\ Olga Swiech, Faculty of Chemistry, University of Warsaw, ul. Pasteura 1, 02-093 Warsaw, \\ Poland. Tel./Fax: +48 22 8220211/+48228225996, E-mail: oswiech@ @ chem.uw.edu.pl
}

Kazimierz Chmurski Faculty of Chemistry, University of Warsaw, ul. Pasteura 1, 02-093 Warsaw, Poland. Tel./Fax: +48 22 8220211/+48228225996, E-mail:

chmurkaz@chem.uw.edu.pl,
Renata Bilewicz, Faculty of Chemistry, University of Warsaw, ul. Pasteura 1, 02-093 Warsaw, Poland. Tel./Fax: +48 22 8220211/+48228225996, E-mail: bilewicz@chem.uw.edu.pl, Corresponding Author.

- Abstract. Complexing abilities of $\beta$-cyclodextrin towards anthraquinone derivatives in solution and immobilized on gold surfaces were studied by voltammetry. The association constant of $\beta$-cyclodextrin with 1-aminoanthraquinone in solution was found to be $1.03 \pm 0.05 \cdot 10^{3} \mathrm{M}^{-1}$ hence smaller than with anthraquinone. Capping the surface immobilized $N$-(1-anthraquinone) lipoamide with $\beta$-cyclodextrin lead to the decrease of heterogeneous electron transfer rate constant due to the change of the immediate environment around the electroactive group. To detect interactions of $\beta$-cyclodextrin with a nonelectroactive guest, $N$-(1-adamantane) lipoamide, the cyclodextrin was modified by attachment of an anthraquinone group as the electroactive marker. The appearance of the voltammetric peak corresponding to reduction of the anthraquinone side-group indicated binding of $\beta$-cyclodextrin to the $N$-(1-adamantane) lipoamide self-assembled in a monolayer on the gold electrode.

- keywords . cyclodextrin, adamantane, anthraquinone, self assembled monolayer, association constant

\section{Introduction}

Cyclodextrins, cyclic organic compounds obtained by enzymatic transformation of starch

belong to one of the most intensively investigated classes of "host" molecules in

supramolecular chemistry. The $\beta$-cyclodextrin $(\beta-C D)$ is one of the most abundant natural

oligomers and corresponds to the association of seven glucose units. The hydrophobic cavity and hydrophilic exterior makes the molecule an appropriate host for various guest molecules bound via non covalent bonds to form inclusion complexes [1, 2]. This inclusion ability of cyclodextrins has attracted considerable attention due to applications in drug delivery systems, sensing devices and for the construction of molecular machines, designed to perform tailored mechanical tasks [3-6]. 
Adamantane, an apolar cage hydrocarbon, is one of guest molecules forming strong inclusion complexes with $\beta-\mathrm{CD}$, with equilibrium constant above $10^{4} \mathrm{M}^{-1}$ [7]. Complexation between $\beta-\mathrm{CD}$ and adamantane and its derivatives has been exploited for molecular linking, gene delivery and sensor applications [8,9].

Anthraquinone derivatives are the largest group of naturally occurring quinones. Redox cycling of anthraquinone is supposed to play a important role in the activation of many anthraquinone-based drugs under aerobic conditions [10]. The inclusion complex between anthraquinone and $\beta-\mathrm{CD}$ in aqueous solution was reported by Jiang et al [11]. To our knowledge there are only few report on the complexes of amino derivatives of anthraquinone with $\beta-C D$ and weaker interactions due to the presence of amino group were shown $[12,13]$.

In the present contribution, the complexation of $\beta$-cyclodextrin with 1-aminoanthraquinone (AAQ) in solution and with surface immobilized $N$-(1-anthraquinone) lipoamide (AQ-Lip), were investigated. (Fig. 1a) Since the anthraquinone group is electroactive, voltammetry can be used to follow the complexation reactions. The electrochemical behavior of a non-electroactive guest: $N$-(1-adamantane) lipoamide (AD-Lip) self-assembled in a monolayer on the gold electrode was also studied. (Fig. 1b). The monolayer covered electrode was exposed to the solution of electroactive derivative of $\beta$-CD: mono-6-deoxy-6-thioureido-(1-anthraquinone)-per-O-methyl- $\beta$-cyclodextrin (AQ- $\beta$-CD) and changes in the voltammograms are discussed in terms of the interaction between the $\beta$-CD and the adamantane moiety.

\section{Experimental}

\section{Chemicals}

All compounds used in this work for the syntheses were purchased from Aldrich and Fluka. $N$-(1-anthraquinone) lipoamide (AQ-Lip). $770 \mathrm{mg}(3.73 \mathrm{mM})$ lipoic acid was mixed with $30 \mathrm{ml}$ of dichloromethane in the reaction vessel. The solution was protected from light, cooled 
to $-1^{\circ} \mathrm{C}$ and kept under argon atmosphere. 1.1 equiv $(4.11 \mathrm{mM} 521 \mathrm{mg})$ of oxalil chloride was added followed by addition of $1 \mathrm{ml}$ of DMF. The reaction mixture was stirred for 3 hours. 1-aminoanthraquinone $(3.8 \mathrm{mM}, 899 \mathrm{mg})$ in $50 \mathrm{ml}$ of dichloromethane was dropped into this mixture and the reaction was continued overnight, while the grey-green precipitate was formed. The latter was filtered and the mixture was evaporated to dryness by means of rotary evaporator. The title compound was isolated by column chromatography on silica gel with dichloromethane as eluent; $\mathrm{Rf}=0.14$. Yield was $342 \mathrm{mg}(0.83 \mathrm{mM})$ as orange solid, $22.3 \%$. MS ES+: AcONa m/z 434.1 [M+Na].

$N$-(1-adamantane) lipoamide (AD-Lip). $2.5 \mathrm{~g}$ of dicyclohexylocarbodiimide (DCC) was dissolved in DMF (8mL). $2.2 \mathrm{~g}(9.8 \mathrm{mM})$ of lipoic acid was added to this solution under magnetic stirring. Immediately, white precipitate was formed. The reaction mixture was diluted with $50 \mathrm{~mL}$ of acetonitrile. To this suspension 1 equiv $(1.86 \mathrm{~g})$ of 1 -amino adamantane hydrochloride was added followed by addition of $1 \mathrm{~mL}$ of triethylamine and the reaction was continued overnight. The precipitate, N,N'-dicyclohexylurea was filtered off and all solvents were removed under reduced pressure. The solid residue was analysed by TLC on silica gel with $5 \% \mathrm{MeOH}$ in dichloromethane as eluent, new compound was detected; $\mathrm{Rf}=0.16$. This product was purified by column chromatography on silica gel with 4:1 v/v chloroform: acetone system as eluent; $\mathrm{Rf}=0.15$. Yield was $1.6 \mathrm{~g}(4.7 \mathrm{mM})$ as yellow solid, $48 \%$. MS ES+ $\mathrm{m} / \mathrm{z} 362.1[\mathrm{M}+\mathrm{Na}]$.

\section{Mono(6-deoxy-6-thioureido(1-antraquinono))-per(2,3,6-O-metylo)- $\beta$-cyclodextrin (AQ- $\boldsymbol{\beta}$-CD). $257 \mathrm{mg}$ (0.246 mM) of mono(6-amino-6-deoxy)-per(2,3,6-O-metylo) $\beta$-cyclodextrin was dissolved in dry pyridine $(5 \mathrm{~mL})$ and 1 equiv of 1-isothiocynatoanthraquinone dissolved in the same solvent was added to this solution at room temperature. After 16 hours, pyridine was evaporated using a rotary evaporator. Remaining traces of pyridine were removed by coevaporation with toluene. Solid residue was dissolved in dichlorometane and purified by}




\section{Electrochemistry}

Electrochemical measurements were performed using a PGSTAT Autolab (Eco Chemie BV, Utrecht, Netherlands). All electrochemical experiments were done in a three-electrode arrangement with silver/silver chloride $(\mathrm{Ag} / \mathrm{AgCl})$ electrode (saturated solution of $\mathrm{KCl})$ as the reference, platinum foil as the counter and Au electrode (BAS, 2 mm diameter) as the working electrode. The working electrode was polished mechanically with 1.0, 0.3 and 0.05 $\mu \mathrm{m}$ alumina powder on a Buehler polishing cloth. Prior to measurements, buffer solutions were purged with purified nitrogen for $30 \mathrm{~min}$ and all experiments were performed at room temperature. Milli-Q ultra-pure water (resistivity $18.2 \mathrm{M} \Omega / \mathrm{cm}$ ) was used.

\section{Preparation of the modified gold electrodes}

The gold electrode was polished to mirror finish with $0.05 \mu \mathrm{m}$ alumina powder and electrochemically cleaned by cycling in the range of potentials from $-0.2 \mathrm{~V}$ to $1.6 \mathrm{~V}$ in $0.5 \mathrm{M}$ $\mathrm{H}_{2} \mathrm{SO}_{4}$ solution until the typical cyclic voltammogram of a clean gold surface was obtained [14]. Modification of the gold electrodes was carried out by self-assembly from oxygen-free 0.1mM solutions of AQ-Lip and AD-Lip in DMF for 25 minutes. Next, the electrodes were immersed in $0.1 \mathrm{mM}$ solution of hexanethiol in DMF for 24 hours. The modified electrode was then washed with Milli-Q ultra-pure water. 


\section{Results and discussion}

\section{B-cyclodextrin complex formation with 1-aminoanthraquinone in solution}

In phosphate buffer with 40 percent DMF ( $\mathrm{pH} 9.1$ ), the decrease of anodic and cathodic peaks of AAQ were observed upon addition of $\beta-C D$ to the AAQ solution due to the smaller diffusion coefficient of the $\beta$-CD complex formed compared to the diffusion coefficient of free guest. Dependence of reduction peak current of AAQ on the ratio of $\beta$-CD to AAQ concentrations is shown in Fig. 2.

The formation constant of 1:1 cyclodextrin complex was calculated using Osa equation [15].

$$
D_{o b s}=\frac{\left(D_{f}-D_{o b s}\right)}{K_{s} \cdot[L]}+D_{c},
$$

where $D_{o b s}$ is the observed diffusion coefficient, and $D_{f}$ and $D_{c}$ are diffusion coefficients of free guest and inclusion complex, respectively. $\mathrm{K}_{\mathrm{s}}$ is formation constant and $[\mathrm{L}]$ is the concentration of the ligand. $\mathrm{D}_{\mathrm{obs}}$ and $\mathrm{D}_{\mathrm{f}}$ can be calculated from the experiments. The value of $K_{s}$ can be obtained from the slope of the linear plot of $D_{\text {obs }} v s .\left(D_{f}-D_{o b s}\right) /[L]$. The formation constant was $1.03 \pm 0.05 \cdot 10^{3} \mathrm{M}^{-1}$.

The ratio of association constants of the reduced and oxidized forms of AAQ are described by equation [3]:

$$
K_{S 1} / K_{S 2}=e^{\left[-F\left(E_{F}^{\prime}-E_{c}^{\prime}\right) / R T\right]}
$$

where, $\mathrm{K}_{\mathrm{S} 1}$ and $\mathrm{K}_{\mathrm{S} 2}$ are the association constants of the oxidized and reduced forms, respectively, and $\mathrm{E}_{\mathrm{F}}$ and $\mathrm{E}_{\mathrm{C}}$ are the formal potentials of free, and complexed forms, respectively. While the peak-to-peak separation increased upon addition of $\beta$-cyclodextrin, the formal potential did not change. In a 1:1 complex this indicated similar binding strength of $\beta$-cyclodextrin with the oxidized and reduced forms of AAQ.

The heterogeneous standard rate constant was calculated from equation [16]: 
Where $\Psi$ is a function fixed from product of electron number $(n)$ and difference between anodic and cathodic peaks potential $\left(E_{o x}-E_{r e d}\right)$. The dependence $\Psi=n\left(E_{o x}-E_{r e d}\right)$ is tabulated. $\mathrm{D}_{\mathrm{ox}}$ and $\mathrm{D}_{\text {red }}$ are diffusion coefficients of anodic and cathodic processes, $\alpha$ is a transfer coefficient, $\mathrm{k}_{\mathrm{s}}$ is heterogeneous rate constants, $\mathrm{v}, \mathrm{R}, \mathrm{T}, \mathrm{F}, \pi$ are their usual meanings

The rate constant of the AAQ electrode process decreases upon addition of $\beta$ cyclodextrin. The values of standard rate constants are $2.5 \cdot 10^{-3} \mathrm{~cm} / \mathrm{s}$ and $0.5 \cdot 10^{-3} \mathrm{~cm} / \mathrm{s}$ for AAQ and AAQ: $\beta-C D$ system, respectively.

The complexation of AAQ by $\beta-C D$ was confirmed using UV-Vis spectrometry. The addition of $\beta-C D$ to the solution of AAQ resulted in the increase of AAQ absorbance

\section{$\beta$-cyclodextrin complex formation with AQ-Lip immobilized in a mixed monolayer at gold electrode}

Two-component monolayers containing AQ-Lip and hexanethiol showed a pair of reversible redox peaks; its anodic and cathodic peak potential where, respectively, $-0.637 \mathrm{~V}$ and $-0.654 \mathrm{~V}$ at $0.05 \mathrm{~V} / \mathrm{s}$ scan rate. The cathodic and anodic peaks were almost symmetric and the formal potential is $-0.645 \mathrm{~V}$ (Fig. 3). The dependence of peak current, $i_{p}$ on scan rate, $v$ is linear and $i_{p}$ is related to surface concentration of the electroactive component of the monolayer, $\Gamma$ according to equation [17]:

$$
i_{p}=\frac{n^{2} \cdot F^{2} \cdot v \cdot A \cdot \Gamma}{4 \cdot R \cdot T}
$$

The surface concentration and molecular area of AQ-Lip modified electrodes calculated based on this equation were $7.73 \pm 0.39 \cdot 10^{-11} \mathrm{~mol} / \mathrm{cm}^{2}$ and $217 \pm 13 \AA^{2}$.

Electrochemical desorption experiments were performed in $0.1 \mathrm{M} \mathrm{NaOH}$ aqueous solution and the surface concentration of the thiolated molecules ( both components of the monolayer) was found to be $3.50 \pm 0.17 \cdot 10^{-10} \mathrm{~mol} / \mathrm{cm}^{2}$. The ratio of surface concentrations can 
be calculated based on these two measurements. For the two-component monolayer AQLip:hexanethiol was 1:4.

The apparent rate constant, $k_{a p p}$ was obtained using equation [18]:

$$
i=k_{a p p} Q \exp \left(-k_{a p p} t\right)
$$

where $\mathrm{Q}$ is the charge associated with converting the redox centers from one oxidation state to another.

The plot of $\ln (\mathrm{i})$ vs. time is linear. The experimental Tafel plot was fitted to theoretical line of the Butler-Volmer equations for low overpotentials region [19]:

$$
\begin{aligned}
& k_{o x}=k_{E T} \exp \left[-\frac{\lambda-2 e_{0} \eta}{4 k_{B} T}\right] \\
& k_{\text {red }}=k_{E T} \exp \left[-\frac{\lambda+2 e_{0} \eta}{4 k_{B} T}\right]
\end{aligned}
$$

where $k_{E T}$ is electron transfer rate at zero overpotential, $\mathrm{k}_{\mathrm{ox}}$ and $\mathrm{k}_{\mathrm{red}}$ are the apparent rate constants for anodic and cathodic processes, $\lambda$ is reorganization energy, $\mathrm{e}_{0}$ and $\mathrm{k}_{\mathrm{B}}$ are static dielectric and Boltzmann constants, and $\eta$ is the applied overpotential .

The dependencies of $\ln \mathrm{k}_{\text {app }}$ vs $\eta$ for mixed AQ-Lip - hexanethiol monolayer in the presence and absence of $\beta-C D$ are shown in Fig. 4. The value of standard rate constant was found to be: 44.1 $\pm 1.7 \mathrm{~s}^{-1}$ without $\beta-\mathrm{CD}$, while in solutions containing $0.1 \mathrm{mM} \beta-\mathrm{CD}$ it decreased to $31.2 \pm 0.8 \mathrm{~s}^{-1}$ In the presence of larger amounts of DMF, the rate constants decreased probably reflecting the interaction with the solvent and a more complicated mechanism. Practically, lack of differences upon addition of $\beta-C D$ to solutions containing DMF may reflect weaker affinity of the $\beta$-CD cavity to AQ-Lip in solutions containing DMF.

In case of adamantane - $\beta$-cyclodextrin complexes both the host and the guest are nonelectroactive and a different method should be used for monitoring the complexation reaction. Our approach was to "decorate" $\beta-C D$ with a side - group which is electroactive. Therefore, $\beta$-cyclodextrin with an anthraquinone side - group was synthesized and its 
electrochemical properties were studied in solution (Fig. 5). The voltammogram showed a cathodic peak at $-0.617 \mathrm{~V}$. The plot of the reduction peak current vs. square root of scan rate is shown in Fig. 6. Positive deviations from linearity at larger scan rates, can be explained by the contribution of adsorption of AQ- $\beta$-CD molecules on the electrode surface.

Since AD-Lip is nonelectroactive, the surface concentration of modified electrodes was calculated from electrochemical desorption of mixed AD-Lip - hexanethiol monolayer in $0.1 \mathrm{M} \mathrm{NaOH}$. The surface concentration of the thiolated molecules in the mixed monolayer was $4.55 \pm 0.9 \cdot 10^{-10} \mathrm{~mol} / \mathrm{cm}^{2}$.

In phosphate buffer solution containing 25 percent DMF ( $\mathrm{pH} 8.9$ ), the interaction between mixed AD-Lip - hexanethiol monolayer modified electrode and AQ- $\beta$-CD was easily detected. Fig.7 - curve a shows the CV curves of bare gold electrode. Curve b was recorded using the electrode covered with mixed AD-Lip - hexanethiol modified gold electrode and curve $\mathrm{c}$ shows, for comparison, the behavior of the AQ- $\beta$-CD system in singlecomponent hexanethiol monolayer. Interaction between bare gold electrode and AQ- $\beta-C D$ leads to the appearance of a cathodic peak, at $-0.777 \mathrm{~V}$. The hexanethiol modified gold electrode exposed to AQ- $\beta-C D$ showed a cathodic peak at potentials $-0.628 \mathrm{~V}$. Finally, the two-component monolayer containing both hexanethiol and Ad-Lip immersed in the AQ- $\beta$ CD solution leads to the appearance of two peaks. Thus, AQ- $\beta-C D$ can affect AD-Lip monolayer in two ways. Firstly, the molecule can be incorporated between other molecules of the monolayer and interact with the electrode surface. This results in the formation of a peak at potential ca. $-0.628 \mathrm{~V}$. In addition, the molecule interacts directly with the AD-Lip component of the monolayer giving the other peak at ca. $-0.735 \mathrm{~V}$.

The peak at $-0.735 \mathrm{~V}$ remains when the electrode is replaced to a clean supporting electrolyte solution and proves the specific interaction of the cyclodextrin with the AD-Lip component of the monolayer. 


\section{Conclusion}

Interaction of $\beta$-cyclodextrin with 1-aminoanthraquinone in the solution and with surface immobilized $N$-(1-anthraquinone) lipoamide slows down the rate of anthraquinone group reduction. In case of solution resident complex, the association constant can be easily evaluated based on the decrease of diffusion coefficient of the electroactive guest due to complexation. The association constant with 1 -aminoanthraquinone was $1.03 \pm 0.05 \cdot 10^{3} \mathrm{M}^{-1}$ hence smaller than that of anthraquinone equal to $2.86 \cdot 10^{3} \mathrm{M}^{-1}$ [20].

The decrease of the electron transfer rate constants of the electroactive anthraquinone moiety upon complexation can be ascribed to the change of its immediate environment caused by the hydrophobicity of the $\beta-\mathrm{CD}$ cavity.

Surface immobilized non-electroactive guest $N$-(1-adamantane) lipoamide was also found to bind $\beta-\mathrm{CD}$ from the solution. The monolayer covered electrode was exposed to the solution of electroactive derivative of $\beta$-CD: mono-6-deoxy-6-thioureido-(1-anthraquinone)per-O-methyl- $\beta$-cyclodextrin (AQ- $\beta$-CD) and then transferred to a pure supporting electrolyte solution. The cyclodextrin was modified by attachment of the anthraquinone group as the electroactive marker. The appearance of the voltammetric peak corresponding to the reduction of the anthraquinone side-group indicated binding of $\beta$-cyclodextrin to the $N$-(1-adamantane) lipoamide monolayer, since it remained upon replacing the modified electrode to the solution of pure supporting electrolyte.

References

1. Szejtli, J. Comprehensive Supramolecular Chemistry, Pergamon: Oxford, 1996.

2. Dodziuk, H. J. Mol Struct, 2002, 614, 33-45.

3. Kaifer, A.E.; Gómez-Kaifer, M. Supramolecular Electrochemistry; Wiley-VCH: Weinheim, 1999.

4. Bilewicz, R Chmurski, K.; in Cyclodextrins and Their Complexes, Chemistry, Analytical Methods, Applications H. Dodziuk (Ed.), Wiley -VCH, Weinheim, 2006, 255-332, 450-474.

5. Chmurski, K.; Temeriusz, A.; Bilewicz, R. Anal. Chem. 2003, 75, 5687-5691.

6. Majewska U.E.; Chmurski, K.; Biesiada, K.; Olszyna, A.; Bilewicz, R. Electroanal. 2006, 18, 1463-1470.

7. Rekharsky, M.V.; Inoue, Y. Chem. Rev. 1998, 98, 1875-1917. 
8. Bellocq, N.C.; Pun, S.H.; Jensen, G.S.; Davis, M.E. Bioconjug. Chem. 2003, 14, 1122-1132.

9. David, C.; Mollot, M.C.; Renard, E.; Sebille, B. J. Incl. Phenom. Macrocycl. Chem. 2002, 44, 369-372.

10. Bachur, N.R.; Gordon, S.L.; Gee. M.V. Cancer Res. 1978, 38, 1745-1750.

11. Jiang, H.; Sun, H.; Zhang, S.; Hua, R.; Xu, Y.; Jin, S.; Gong, H.; Li, L. J. Incl. Phenom. Macrocycl. Chem. 2007, 58, 133-138.

12. Shamsipur, M.; Yari, A.; Sharghi, H. Spectrochim. Acta A, 2005, 62, 372-376.

13. Garcia Sanchez, F.; Lopez, M.H.; De Garcia Villodres, E. Mikrochim. Acta 1987, 2, 217-224

14. Hoare, J.P. J. Electrochem. Soc. 1984, 131, 1808-1812.

15. Osa, T.; Matsue, T.; Fujihira, T. Heterocycles, 1977, 6, 1833-1839.

16. Nicholson, R.S. Anal. Chem. 1965, 37, 1351-1355.

17. Laviron, E.J. Electroanal. Chem. 1974, 52, 355-393.

18. Finklea, H.O.; Hanshew, D.D. J. Am. Chem. Soc. 1992, 114, 3173-3181.

19. Eckermann, A.L.; Feld, D.J.; Shaw, J.A.; Meade, T.J. Coord. Chem. Rev. 2008 doi:10.1016/j.ccr.2009.12.023

20. Dang, X.J.; Tong, J.; Li, H.L. J. Incl. Phen. Macrocycl. Chem. 1996, 24, 275-286. 
Figure 1. Structures of AQ-Lip(a) and AD-Lip(b).

Figure 2. Dependence of reduction peak current of 1-aminoanthraquinone on the ratio of concentrations of cyclodextrin to 1-aminoanthraquinone.

Figure 3. Cyclic voltammogram of mixed AQ-Lip-hexanethiol modified gold electrode performed in phosphate buffer with $40 \%$ addition of DMF. Scan rate 50 $\mathrm{mV} / \mathrm{s}$.

Figure 4. Tafel plot for mixed AQ-Lip - hexanethiol monolayer in the presence and absence of $\beta$-CD recorded in 0.1 M phosphate buffer without DMF, $\mathrm{pH} 9.1$.

Figure 5. Cyclic voltammogram of AQ- $\beta-C D$ in phosphate buffer solution with $25 \%$ DMF. Scan rate $50 \mathrm{mV} / \mathrm{s}$.

Figure 6 . The dependence of cathodic peak current on scan rate for AQ- $\beta$-CD solution. Supporting electrolyte: $0.1 \mathrm{M}$ phosphate buffer $+25 \%$ DMF, pH 8.9. Figure 7. Cyclic voltammograms recorded using a (a) gold electrode and electrode modified by: (b) mixture of $N$-(1-adamantane) lipoamide and hexanethiol, (c) hexanethiol, in phosphate buffer containing 25\% DMF, $\mathrm{pH}$ 8.9. All electrodes were kept in $0.1 \mathrm{mM}$ solution of mono-6-deoxy-6-thioureido-(1-anthraquinone)-per-Omethyl- $\beta$-cyclodextrin for two hours. 


\section{Molecular Interactions of $\beta$-Cyclodextrins with Monolayers Containing Adamantane and Anthraquinone Guest Groups}

\section{Olga Swiech, Kazimierz Chmurski, Renata Bilewicz*}

Department of Chemistry, University of Warsaw, Pasteura 1, 02-093 Warsaw, Poland

Olga Swiech, Faculty of Chemistry, University of Warsaw, ul. Pasteura 1, 02-093 Warsaw, Poland. Tel./Fax: +48 22 8220211/+48228225996, E-mail: oswiech@chem.uw.edu.pl

Kazimierz Chmurski Faculty of Chemistry, University of Warsaw, ul. Pasteura 1, 02-093 Warsaw, Poland. Tel./Fax: +48 22 8220211/+48228225996, E-mail: chmurkaz@chem.uw.edu.pl,

Renata Bilewicz, Faculty of Chemistry, University of Warsaw, ul. Pasteura 1, 02-093 Warsaw, Poland. Tel./Fax: +48 22 8220211/+48228225996, E-mail: bilewicz@chem.uw.edu.pl, Corresponding Author.

- Abstract. Complexing abilities of $\beta$-cyclodextrin towards anthraquinone derivatives in solution and immobilized on gold surfaces were studied by voltammetry. The association constant of $\beta$-cyclodextrin with 1-aminoanthraquinone in solution was found to be $1.03 \pm 0.05 \cdot 10^{3} \mathrm{M}^{-1}$ hence smaller than with anthraquinone. Capping the surface immobilized $N$-(1-anthraquinone) lipoamide with $\beta$-cyclodextrin lead to the decrease of heterogeneous electron transfer rate constant due to the change of the immediate environment around the electroactive group. To detect interactions of $\beta$-cyclodextrin with a nonelectroactive guest, $N$-(1-adamantane) lipoamide, the cyclodextrin was modified by attachment of an anthraquinone group as the electroactive marker. The appearance of the voltammetric peak corresponding to reduction of the anthraquinone side-group indicated binding of $\beta$-cyclodextrin to the $N$-(1-adamantane) lipoamide self-assembled in a monolayer on the gold electrode.

- keywords . cyclodextrin, adamantane, anthraquinone, self assembled monolayer, association constant

\section{Introduction}

Cyclodextrins, cyclic organic compounds obtained by enzymatic transformation of starch

belong to one of the most intensively investigated classes of "host" molecules in

supramolecular chemistry. The $\beta$-cyclodextrin $(\beta-C D)$ is one of the most abundant natural

oligomers and corresponds to the association of seven glucose units. The hydrophobic cavity and hydrophilic exterior makes the molecule an appropriate host for various guest molecules bound via non covalent bonds to form inclusion complexes [1,2]. This inclusion ability of cyclodextrins has attracted considerable attention due to applications in drug delivery systems, sensing devices and for the construction of molecular machines, designed to perform tailored mechanical tasks [3-6]. 
Adamantane, an apolar cage hydrocarbon, is one of guest molecules forming strong inclusion complexes with $\beta-\mathrm{CD}$, with equilibrium constant above $10^{4} \mathrm{M}^{-1}$ [7]. Complexation between $\beta-\mathrm{CD}$ and adamantane and its derivatives has been exploited for molecular linking, gene delivery and sensor applications $[8,9]$.

Anthraquinone derivatives are the largest group of naturally occurring quinones. Redox cycling of anthraquinone is supposed to play a important role in the activation of many anthraquinone-based drugs under aerobic conditions [10]. The inclusion complex between anthraquinone and $\beta-\mathrm{CD}$ in aqueous solution was reported by Jiang et al [11]. To our knowledge there are only few report on the complexes of amino derivatives of anthraquinone with $\beta-C D$ and weaker interactions due to the presence of amino group were shown $[12,13]$.

In the present contribution, the complexation of $\beta$-cyclodextrin with 1-aminoanthraquinone (AAQ) in solution and with surface immobilized $N$-(1-anthraquinone) lipoamide (AQ-Lip), were investigated. (Fig. 1a) Since the anthraquinone group is electroactive, voltammetry can be used to follow the complexation reactions. The electrochemical behavior of a non-electroactive guest: $N$-(1-adamantane) lipoamide (AD-Lip) self-assembled in a monolayer on the gold electrode was also studied. (Fig. 1b). The monolayer covered electrode was exposed to the solution of electroactive derivative of $\beta-\mathrm{CD}$ : mono-6-deoxy-6-thioureido-(1-anthraquinone)-per-O-methyl- $\beta$-cyclodextrin (AQ- $\beta$-CD) and changes in the voltammograms are discussed in terms of the interaction between the $\beta$-CD and the adamantane moiety.

\section{Experimental}

\section{Chemicals}

All compounds used in this work for the syntheses were purchased from Aldrich and Fluka. N-(1-anthraquinone) lipoamide (AQ-Lip). $770 \mathrm{mg}(3.73 \mathrm{mM})$ lipoic acid was mixed with $30 \mathrm{ml}$ of dichloromethane in the reaction vessel. The solution was protected from light, cooled 
to $-1^{\circ} \mathrm{C}$ and kept under argon atmosphere. 1.1 equiv $(4.11 \mathrm{mM} 521 \mathrm{mg}$ ) of oxalil chloride was added followed by addition of $1 \mathrm{ml}$ of DMF. The reaction mixture was stirred for 3 hours. 1-aminoanthraquinone $(3.8 \mathrm{mM}, 899 \mathrm{mg})$ in $50 \mathrm{ml}$ of dichloromethane was dropped into this mixture and the reaction was continued overnight, while the grey-green precipitate was formed. The latter was filtered and the mixture was evaporated to dryness by means of rotary evaporator. The title compound was isolated by column chromatography on silica gel with dichloromethane as eluent; $\mathrm{Rf}=0.14$. Yield was $342 \mathrm{mg}(0.83 \mathrm{mM})$ as orange solid, $22.3 \%$. MS ES+: AcONa m/z 434.1 [M+Na].

$N$-(1-adamantane) lipoamide (AD-Lip). $2.5 \mathrm{~g}$ of dicyclohexylocarbodiimide (DCC) was dissolved in DMF (8mL). $2.2 \mathrm{~g}(9.8 \mathrm{mM})$ of lipoic acid was added to this solution under magnetic stirring. Immediately, white precipitate was formed. The reaction mixture was diluted with $50 \mathrm{~mL}$ of acetonitrile. To this suspension 1 equiv $(1.86 \mathrm{~g})$ of 1 -amino adamantane hydrochloride was added followed by addition of $1 \mathrm{~mL}$ of triethylamine and the reaction was continued overnight. The precipitate, N,N'-dicyclohexylurea was filtered off and all solvents were removed under reduced pressure. The solid residue was analysed by TLC on silica gel with $5 \% \mathrm{MeOH}$ in dichloromethane as eluent, new compound was detected; $\mathrm{Rf}=0.16$. This product was purified by column chromatography on silica gel with 4:1 v/v chloroform: acetone system as eluent; $\mathrm{Rf}=0.15$. Yield was $1.6 \mathrm{~g}(4.7 \mathrm{mM})$ as yellow solid, $48 \%$. MS ES+ $\mathrm{m} / \mathrm{z} 362.1[\mathrm{M}+\mathrm{Na}]$.

\section{Mono(6-deoxy-6-thioureido(1-antraquinono))-per(2,3,6- $O$-metylo)- $\beta$-cyclodextrin (AQ-} $\boldsymbol{\beta}$-CD). $257 \mathrm{mg}(0.246 \mathrm{mM})$ of mono(6-amino-6-deoxy)-per(2,3,6-O-metylo) $\beta$-cyclodextrin was dissolved in dry pyridine $(5 \mathrm{~mL})$ and 1 equiv of 1-isothiocynatoanthraquinone dissolved in the same solvent was added to this solution at room temperature. After 16 hours, pyridine was evaporated using a rotary evaporator. Remaining traces of pyridine were removed by coevaporation with toluene. Solid residue was dissolved in dichlorometane and purified by 
column chromatography on silica gel with $5 \% \mathrm{MeOH}$ in dichlorometane as eluent; $\mathrm{Rf}=0.2$. Orange amorphous solid was obtained with yield $300 \mathrm{mg}(0.229 \mathrm{mM})$ 92,9\%. MS TOF $\mathrm{ES}+\mathrm{m} / \mathrm{z} 1719[\mathrm{M}+\mathrm{Na}]$. NMR revealed loss of symmetry of the macrocycle resulting in signal broadening ${ }^{1} \mathrm{H}$ NMR $\left(200 \mathrm{MHz}, \mathrm{CD}_{2} \mathrm{Cl}_{2}\right) \delta=8.5-7.56(5 \mathrm{~m}, 7 \mathrm{H})$ anthraquinone, 5.33-5.51 (m, $\left.\mathrm{dxd}, \mathrm{m}, 7 \mathrm{H}) \mathrm{H} 1^{\mathrm{I}}, \mathrm{H} 1^{\mathrm{II}-\mathrm{VII}}\right), 4-3$ (m remaining $\left.\mathrm{H}\right):{ }^{13} \mathrm{C}\left(50,28 \mathrm{MHz} \mathrm{CD}_{2} \mathrm{Cl}_{2}\right) \delta=185.88 \mathrm{C}=\mathrm{O}$, 183.03 CS, 134-120 Anthraquinone aromatic C, 99.48-99.075 C-1, 82.9-78.72 C-4, 72.3970.68 C-2, C-3, C-5, 60.03-58.31 C3-OMe , 54.92-52.76 C2-OMe, C6-OMe

\section{Electrochemistry}

Electrochemical measurements were performed using a PGSTAT Autolab (Eco Chemie BV, Utrecht, Netherlands). All electrochemical experiments were done in a three-electrode arrangement with silver/silver chloride $(\mathrm{Ag} / \mathrm{AgCl})$ electrode (saturated solution of $\mathrm{KCl})$ as the reference, platinum foil as the counter and Au electrode (BAS, 2 mm diameter) as the working electrode. The working electrode was polished mechanically with 1.0, 0.3 and 0.05 $\mu \mathrm{m}$ alumina powder on a Buehler polishing cloth. Prior to measurements, buffer solutions were purged with purified nitrogen for $30 \mathrm{~min}$ and all experiments were performed at room temperature. Milli-Q ultra-pure water (resistivity $18.2 \mathrm{M} \Omega / \mathrm{cm}$ ) was used.

\section{Preparation of the modified gold electrodes}

The gold electrode was polished to mirror finish with $0.05 \mu \mathrm{m}$ alumina powder and electrochemically cleaned by cycling in the range of potentials from $-0.2 \mathrm{~V}$ to $1.6 \mathrm{~V}$ in $0.5 \mathrm{M}$ $\mathrm{H}_{2} \mathrm{SO}_{4}$ solution until the typical cyclic voltammogram of a clean gold surface was obtained [14]. Modification of the gold electrodes was carried out by self-assembly from oxygen-free 0.1mM solutions of AQ-Lip and AD-Lip in DMF for 25 minutes. Next, the electrodes were immersed in $0.1 \mathrm{mM}$ solution of hexanethiol in DMF for 24 hours. The modified electrode was then washed with Milli-Q ultra-pure water. 


\section{Results and discussion}

\section{B-cyclodextrin complex formation with 1-aminoanthraquinone in solution}

In phosphate buffer with 40 percent DMF ( $\mathrm{pH} 9.1$ ), the decrease of anodic and cathodic peaks of AAQ were observed upon addition of $\beta-C D$ to the AAQ solution due to the smaller diffusion coefficient of the $\beta$-CD complex formed compared to the diffusion coefficient of free guest. Dependence of reduction peak current of AAQ on the ratio of $\beta$-CD to AAQ concentrations is shown in Fig. 2.

The formation constant of 1:1 cyclodextrin complex was calculated using Osa equation [15].

$$
D_{o b s}=\frac{\left(D_{f}-D_{o b s}\right)}{K_{s} \cdot[L]}+D_{c},
$$

where $D_{o b s}$ is the observed diffusion coefficient, and $D_{f}$ and $D_{c}$ are diffusion coefficients of free guest and inclusion complex, respectively. $\mathrm{K}_{\mathrm{s}}$ is formation constant and $[\mathrm{L}]$ is the concentration of the ligand. $D_{o b s}$ and $D_{f}$ can be calculated from the experiments. The value of $K_{s}$ can be obtained from the slope of the linear plot of $D_{\text {obs }}$ vs. $\left(D_{f}-D_{o b s}\right) /[L]$. The formation constant was $1.03 \pm 0.05 \cdot 10^{3} \mathrm{M}^{-1}$.

The ratio of association constants of the reduced and oxidized forms of AAQ are described by equation [3]:

$$
K_{S 1} / K_{S 2}=e^{\left[-F\left(E_{F}^{\prime}-E_{c}^{\prime}\right) / R T\right]},
$$

where, $\mathrm{K}_{\mathrm{S} 1}$ and $\mathrm{K}_{\mathrm{S} 2}$ are the association constants of the oxidized and reduced forms, respectively, and $\mathrm{E}_{\mathrm{F}}$ and $\mathrm{E}_{\mathrm{C}}$ are the formal potentials of free, and complexed forms, respectively. While the peak-to-peak separation increased upon addition of $\beta$-cyclodextrin, the formal potential did not change. In a 1:1 complex this indicated similar binding strength of $\beta$-cyclodextrin with the oxidized and reduced forms of AAQ.

The heterogeneous standard rate constant was calculated from equation [16]: 


$$
\Psi=\left(\frac{D_{o x}}{D_{\text {red }}}\right)^{\alpha / 2} \frac{k_{s}(R T)^{1 / 2}}{\left(\pi n F v D_{o x}\right)^{1 / 2}}
$$

Where $\Psi$ is a function fixed from product of electron number $(n)$ and difference between anodic and cathodic peaks potential $\left(E_{o x}-E_{r e d}\right)$. The dependence $\Psi=n\left(E_{o x}-E_{r e d}\right)$ is tabulated. $\mathrm{D}_{\mathrm{ox}}$ and $\mathrm{D}_{\text {red }}$ are diffusion coefficients of anodic and cathodic processes, $\alpha$ is a transfer coefficient, $\mathrm{k}_{\mathrm{s}}$ is heterogeneous rate constants, $\mathrm{v}, \mathrm{R}, \mathrm{T}, \mathrm{F}, \pi$ are their usual meanings

The rate constant of the AAQ electrode process decreases upon addition of $\beta$ cyclodextrin. The values of standard rate constants are $2.5 \cdot 10^{-3} \mathrm{~cm} / \mathrm{s}$ and $0.5 \cdot 10^{-3} \mathrm{~cm} / \mathrm{s}$ for AAQ and AAQ: $\beta-C D$ system, respectively.

The complexation of AAQ by $\beta-C D$ was confirmed using UV-Vis spectrometry. The addition of $\beta-C D$ to the solution of AAQ resulted in the increase of AAQ absorbance

\section{$\beta$-cyclodextrin complex formation with AQ-Lip immobilized in a mixed monolayer at gold electrode}

Two-component monolayers containing AQ-Lip and hexanethiol showed a pair of reversible redox peaks; its anodic and cathodic peak potential where, respectively, $-0.637 \mathrm{~V}$ and $-0.654 \mathrm{~V}$ at $0.05 \mathrm{~V} / \mathrm{s}$ scan rate. The cathodic and anodic peaks were almost symmetric and the formal potential is $-0.645 \mathrm{~V}$ (Fig. 3). The dependence of peak current, $i_{p}$ on scan rate, $v$ is linear and $i_{p}$ is related to surface concentration of the electroactive component of the monolayer, $\Gamma$ according to equation [17]:

$$
i_{p}=\frac{n^{2} \cdot F^{2} \cdot v \cdot A \cdot \Gamma}{4 \cdot R \cdot T}
$$

The surface concentration and molecular area of AQ-Lip modified electrodes calculated based on this equation were $7.73 \pm 0.39 \cdot 10^{-11} \mathrm{~mol} / \mathrm{cm}^{2}$ and $217 \pm 13 \AA^{2}$.

Electrochemical desorption experiments were performed in $0.1 \mathrm{M} \mathrm{NaOH}$ aqueous solution and the surface concentration of the thiolated molecules ( both components of the monolayer) was found to be $3.50 \pm 0.17 \cdot 10^{-10} \mathrm{~mol} / \mathrm{cm}^{2}$. The ratio of surface concentrations can 
be calculated based on these two measurements. For the two-component monolayer AQ-

\section{Lip:hexanethiol was 1:4.}

The apparent rate constant, $k_{a p p}$ was obtained using equation [18]:

$$
i=k_{\text {app }} Q \exp \left(-k_{\text {app }} t\right)
$$

where $\mathrm{Q}$ is the charge associated with converting the redox centers from one oxidation state to another.

The plot of $\ln (\mathrm{i})$ vs. time is linear. The experimental Tafel plot was fitted to theoretical line of the Butler-Volmer equations for low overpotentials region [19]:

$$
\begin{aligned}
& k_{o x}=k_{E T} \exp \left[-\frac{\lambda-2 e_{0} \eta}{4 k_{B} T}\right] \\
& k_{\text {red }}=k_{E T} \exp \left[-\frac{\lambda+2 e_{0} \eta}{4 k_{B} T}\right]
\end{aligned}
$$

where $k_{E T}$ is electron transfer rate at zero overpotential, $\mathrm{k}_{\mathrm{ox}}$ and $\mathrm{k}_{\mathrm{red}}$ are the apparent rate constants for anodic and cathodic processes, $\lambda$ is reorganization energy, $\mathrm{e}_{0}$ and $\mathrm{k}_{\mathrm{B}}$ are static dielectric and Boltzmann constants, and $\eta$ is the applied overpotential . The dependencies of $\ln \mathrm{k}_{\mathrm{app}}$ vs $\eta$ for mixed AQ-Lip - hexanethiol monolayer in the presence and absence of $\beta-C D$ are shown in Fig. 4. The value of standard rate constant was found to be: 44.1 $\pm 1.7 \mathrm{~s}^{-1}$ without $\beta-\mathrm{CD}$, while in solutions containing $0.1 \mathrm{mM} \beta-\mathrm{CD}$ it decreased to $31.2 \pm 0.8 \mathrm{~s}^{-1}$ In the presence of larger amounts of DMF, the rate constants decreased probably reflecting the interaction with the solvent and a more complicated mechanism. Practically, lack of differences upon addition of $\beta$-CD to solutions containing DMF may reflect weaker affinity of the $\beta$-CD cavity to AQ-Lip in solutions containing DMF.

In case of adamantane - $\beta$-cyclodextrin complexes both the host and the guest are nonelectroactive and a different method should be used for monitoring the complexation reaction. Our approach was to "decorate" $\beta-\mathrm{CD}$ with a side - group which is electroactive. Therefore, $\beta$-cyclodextrin with an anthraquinone side - group was synthesized and its 
electrochemical properties were studied in solution (Fig. 5). The voltammogram showed a cathodic peak at $-0.617 \mathrm{~V}$. The plot of the reduction peak current vs. square root of scan rate is shown in Fig. 6. Positive deviations from linearity at larger scan rates, can be explained by the contribution of adsorption of AQ- $\beta-C D$ molecules on the electrode surface.

Since AD-Lip is nonelectroactive, the surface concentration of modified electrodes was calculated from electrochemical desorption of mixed AD-Lip - hexanethiol monolayer in $0.1 \mathrm{M} \mathrm{NaOH}$. The surface concentration of the thiolated molecules in the mixed monolayer was $4.55 \pm 0.9 \cdot 10^{-10} \mathrm{~mol} / \mathrm{cm}^{2}$.

In phosphate buffer solution containing 25 percent $\mathrm{DMF}(\mathrm{pH} 8.9)$, the interaction between mixed AD-Lip - hexanethiol monolayer modified electrode and AQ- $\beta$-CD was easily detected. Fig.7 - curve a shows the CV curves of bare gold electrode. Curve b was recorded using the electrode covered with mixed AD-Lip - hexanethiol modified gold electrode and curve $\mathrm{c}$ shows, for comparison, the behavior of the AQ- $\beta$-CD system in singlecomponent hexanethiol monolayer. Interaction between bare gold electrode and AQ- $\beta$-CD leads to the appearance of a cathodic peak, at $-0.777 \mathrm{~V}$. The hexanethiol modified gold electrode exposed to AQ- $\beta$-CD showed a cathodic peak at potentials $-0.628 \mathrm{~V}$. Finally, the two-component monolayer containing both hexanethiol and Ad-Lip immersed in the AQ- $\beta$ CD solution leads to the appearance of two peaks. Thus, AQ- $\beta$-CD can affect AD-Lip monolayer in two ways. Firstly, the molecule can be incorporated between other molecules of the monolayer and interact with the electrode surface. This results in the formation of a peak at potential ca. $-0.628 \mathrm{~V}$. In addition, the molecule interacts directly with the AD-Lip component of the monolayer giving the other peak at ca. $-0.735 \mathrm{~V}$.

The peak at $-0.735 \mathrm{~V}$ remains when the electrode is replaced to a clean supporting electrolyte solution and proves the specific interaction of the cyclodextrin with the AD-Lip component of the monolayer. 


\section{Conclusion}

Interaction of $\beta$-cyclodextrin with 1-aminoanthraquinone in the solution and with surface immobilized $N$-(1-anthraquinone) lipoamide slows down the rate of anthraquinone group reduction. In case of solution resident complex, the association constant can be easily evaluated based on the decrease of diffusion coefficient of the electroactive guest due to complexation. The association constant with 1 -aminoanthraquinone was $1.03 \pm 0.05 \cdot 10^{3} \mathrm{M}^{-1}$ hence smaller than that of anthraquinone equal to $2.86 \cdot 10^{3} \mathrm{M}^{-1}$ [20].

The decrease of the electron transfer rate constants of the electroactive anthraquinone moiety upon complexation can be ascribed to the change of its immediate environment caused by the hydrophobicity of the $\beta-\mathrm{CD}$ cavity.

Surface immobilized non-electroactive guest $N$-(1-adamantane) lipoamide was also found to bind $\beta-\mathrm{CD}$ from the solution. The monolayer covered electrode was exposed to the solution of electroactive derivative of $\beta$-CD: mono-6-deoxy-6-thioureido-(1-anthraquinone)per-O-methyl- $\beta$-cyclodextrin (AQ- $\beta-C D)$ and then transferred to a pure supporting electrolyte solution. The cyclodextrin was modified by attachment of the anthraquinone group as the electroactive marker. The appearance of the voltammetric peak corresponding to the reduction of the anthraquinone side-group indicated binding of $\beta$-cyclodextrin to the $N$-(1-adamantane) lipoamide monolayer, since it remained upon replacing the modified electrode to the solution of pure supporting electrolyte.

\section{References}

1. Szejtli, J. Comprehensive Supramolecular Chemistry, Pergamon: Oxford, 1996.

2. Dodziuk, H. J. Mol Struct, 2002, 614, 33-45.

3. Kaifer, A.E.; Gómez-Kaifer, M. Supramolecular Electrochemistry; Wiley-VCH: Weinheim, 1999.

4. Bilewicz, R Chmurski, K.; in Cyclodextrins and Their Complexes, Chemistry, Analytical Methods, Applications H. Dodziuk (Ed.), Wiley -VCH, Weinheim, 2006, 255-332, 450-474.

5. Chmurski, K.; Temeriusz, A.; Bilewicz, R. Anal. Chem. 2003, 75, 5687-5691.

6. Majewska U.E.; Chmurski, K.; Biesiada, K.; Olszyna, A.; Bilewicz, R. Electroanal. 2006, 18, 1463-1470.

7. Rekharsky, M.V.; Inoue, Y. Chem. Rev. 1998, 98, 1875-1917. 
8. Bellocq, N.C.; Pun, S.H.; Jensen, G.S.; Davis, M.E. Bioconjug. Chem. 2003, 14, 1122-1132.

9. David, C.; Mollot, M.C.; Renard, E.; Sebille, B. J. Incl. Phenom. Macrocycl. Chem. 2002, 44, 369-372.

10. Bachur, N.R.; Gordon, S.L.; Gee. M.V. Cancer Res. 1978, 38, 1745-1750.

11. Jiang, H.; Sun, H.; Zhang, S.; Hua, R.; Xu, Y.; Jin, S.; Gong, H.; Li, L. J. Incl. Phenom. Macrocycl. Chem. 2007, 58, 133-138.

12. Shamsipur, M.; Yari, A.; Sharghi, H. Spectrochim. Acta A, 2005, 62, 372-376.

13. Garcia Sanchez, F.; Lopez, M.H.; De Garcia Villodres, E. Mikrochim. Acta 1987, 2, 217-224

14. Hoare, J.P. J. Electrochem. Soc. 1984, 131, 1808-1812.

15. Osa, T.; Matsue, T.; Fujihira, T. Heterocycles, 1977, 6, 1833-1839.

16. Nicholson, R.S. Anal. Chem. 1965, 37, 1351-1355.

17. Laviron, E.J. Electroanal. Chem. 1974, 52, 355-393.

18. Finklea, H.O.; Hanshew, D.D. J. Am. Chem. Soc. 1992, 114, 3173-3181.

19. Eckermann, A.L.; Feld, D.J.; Shaw, J.A.; Meade, T.J. Coord. Chem. Rev. 2008 doi:10.1016/j.ccr.2009.12.023

20. Dang, X.J.; Tong, J.; Li, H.L. J. Incl. Phen. Macrocycl. Chem. 1996, 24, 275-286. 
Figure 1. Structures of AQ-Lip(a) and AD-Lip(b).

Figure 2. Dependence of reduction peak current of 1-aminoanthraquinone on the ratio of concentrations of cyclodextrin to 1-aminoanthraquinone.

Figure 3. Cyclic voltammogram of mixed AQ-Lip-hexanethiol modified gold electrode performed in phosphate buffer with $40 \%$ addition of DMF. Scan rate: 50 $\mathrm{mV} / \mathrm{s}$.

Figure 4. Tafel plot for mixed AQ-Lip - hexanethiol monolayer in the presence and absence of $\beta$-CD recorded in $0.1 \mathrm{M}$ phosphate buffer without DMF, $\mathrm{pH} 9.1$.

Figure 5. Cyclic voltammogram of AQ- $\beta-C D$ in phosphate buffer solution with $25 \%$ DMF. Scan rate $50 \mathrm{mV} / \mathrm{s}$.

Figure 6. The dependence of cathodic peak current on scan rate for AQ- $\beta-C D$ solution. Supporting electrolyte: $0.1 \mathrm{M}$ phosphate buffer $+25 \%$ DMF, pH 8.9. Figure 7. Cyclic voltammograms recorded using (a) gold electrode and electrode modified by: (b) mixture of $N$-(1-adamantane) lipoamide and hexanethiol, (c) hexanethiol, in phosphate buffer containing $25 \% \mathrm{DMF}, \mathrm{pH}$ 8.9. All electrodes were kept in $0.1 \mathrm{mM}$ solution of mono-6-deoxy-6-thioureido-(1-anthraquinone)-per-Omethyl- $\beta$-cyclodextrin for two hours. 


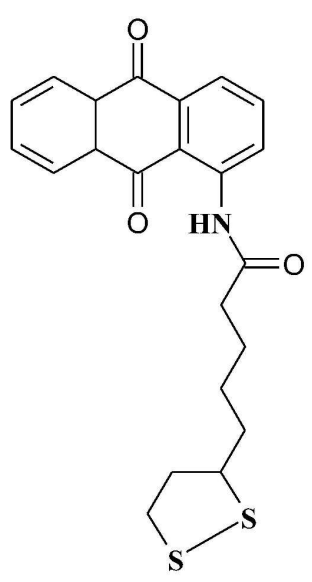

a

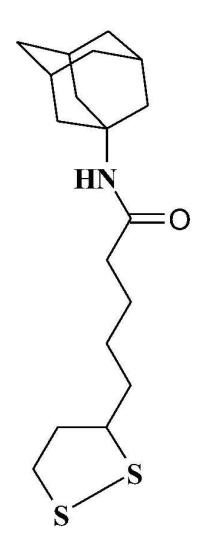

b

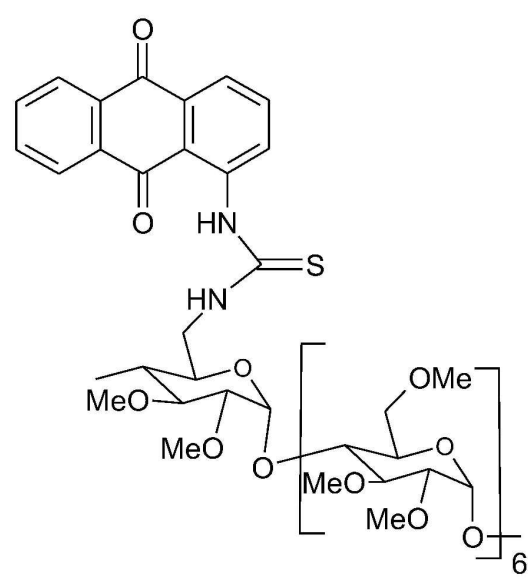

c

Figure 1. Structures of AQ-Lip (a), AD-Lip (b) and AQ- $\beta-C D$ (c) $187 \times 102 \mathrm{~mm}(600 \times 600 \mathrm{DPI})$ 
1

2

3

4

5

6

7

8

9

10

11

12

13

14

15

16

17

18

19

20

21

22

23

24

25

26

27

28

29

30

31

32

33

34

35

36

37

38

39

40

41

42

43

44

45

46

47

48

49

50

51

52

53

54

55

56

57

58

59

60

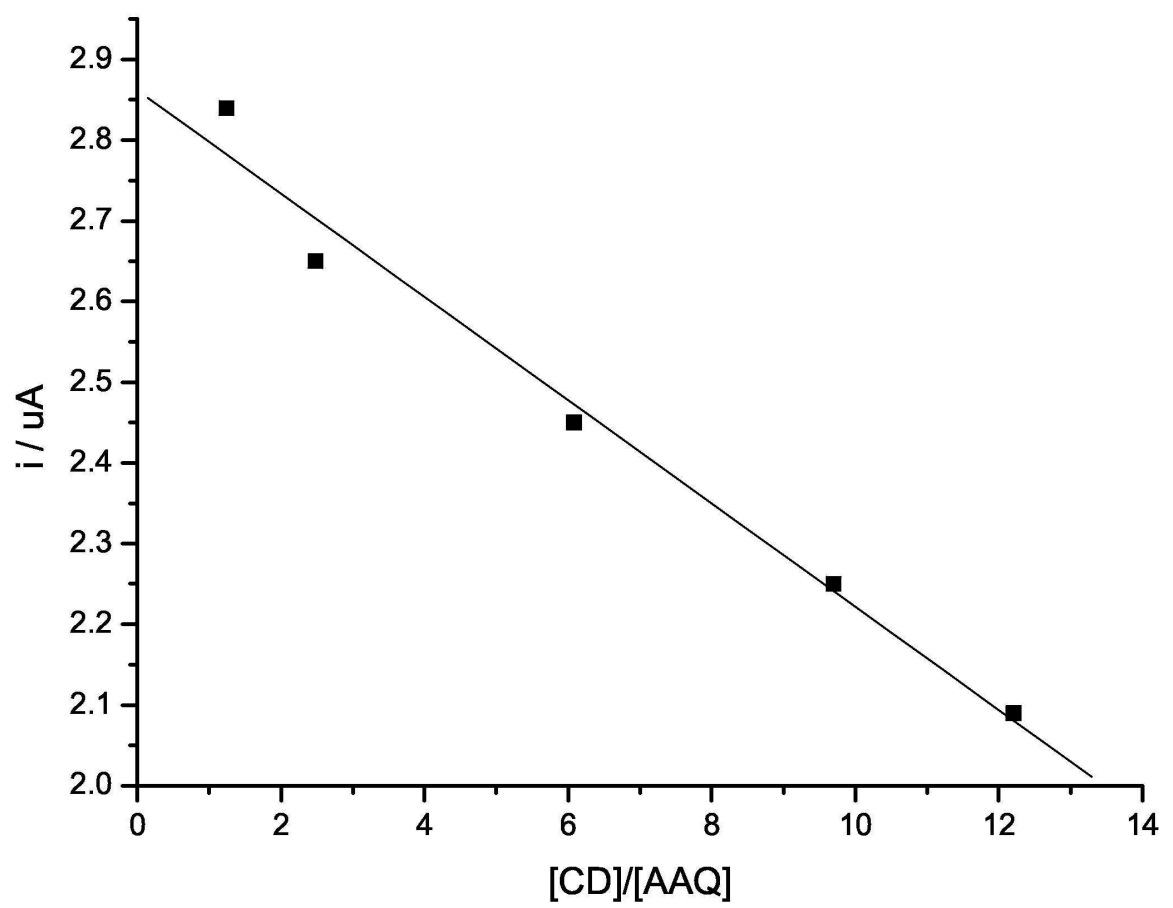

Figure 2. Dependence of reduction peak current of 1-aminoanthraquinone on the ratio of concentrations of cyclodextrin to 1-aminoanthraquinone. $99 \times 79 \mathrm{~mm}(600 \times 600 \mathrm{DPI})$ 
Figure 3. Cyclic voltammogram of mixed AQ-Lip-hexanethiol modified gold electrode performed in phosphate buffer with $40 \%$ addition of DMF. Scan rate: $50 \mathrm{mV} / \mathrm{s}$. $100 \times 80 \mathrm{~mm}(600 \times 600 \mathrm{DPI})$

URL: http:/mc.manuscriptcentral.com/tandf/gsch Email: suprachem@mail.cm.utexas.edu 


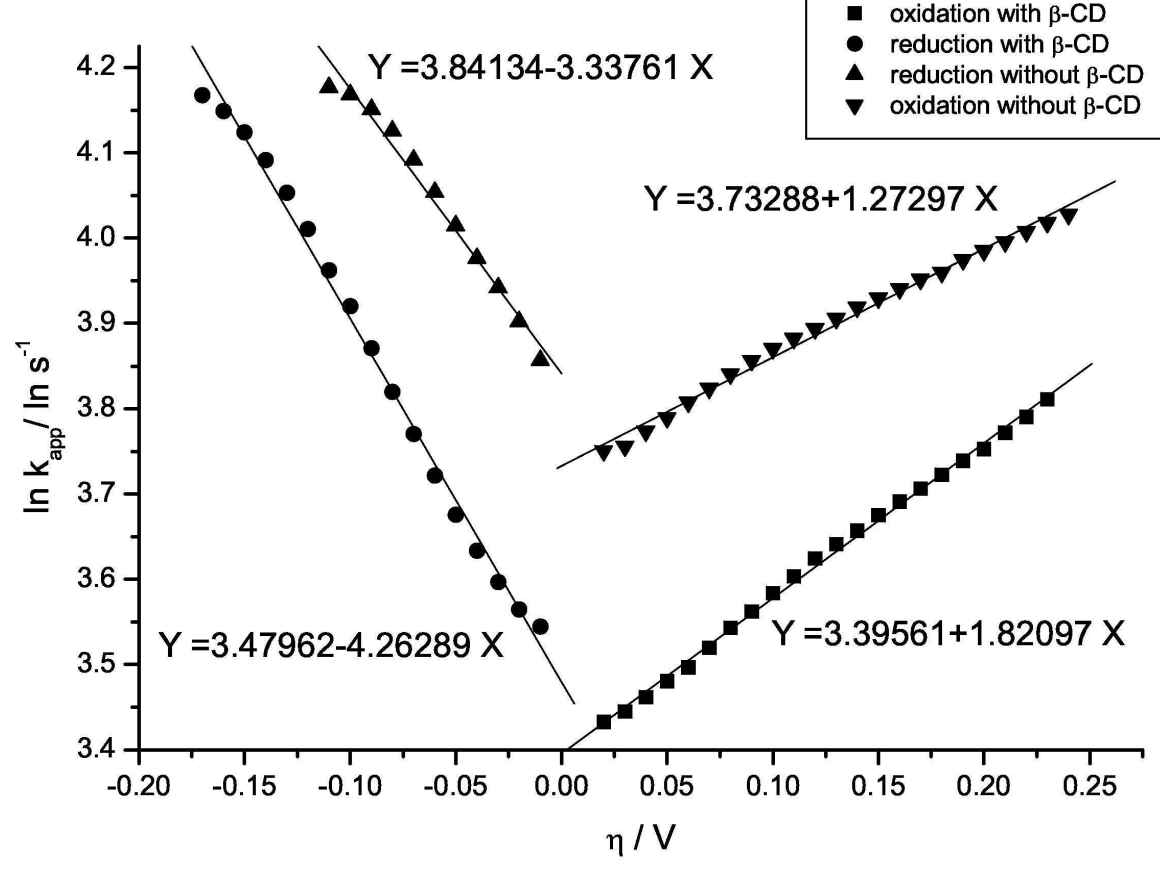

Figure 4. Tafel plot for mixed AQ-Lip - hexanethiol monolayer in the presence and absence of $\beta$-CD recorded in $0.1 \mathrm{M}$ phosphate buffer without DMF, pH 9.1. $107 \times 86 \mathrm{~mm}(600 \times 600 \mathrm{DPI})$ 


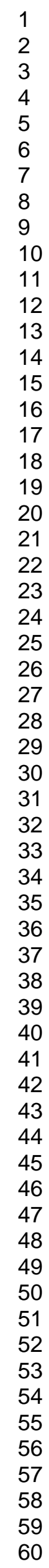

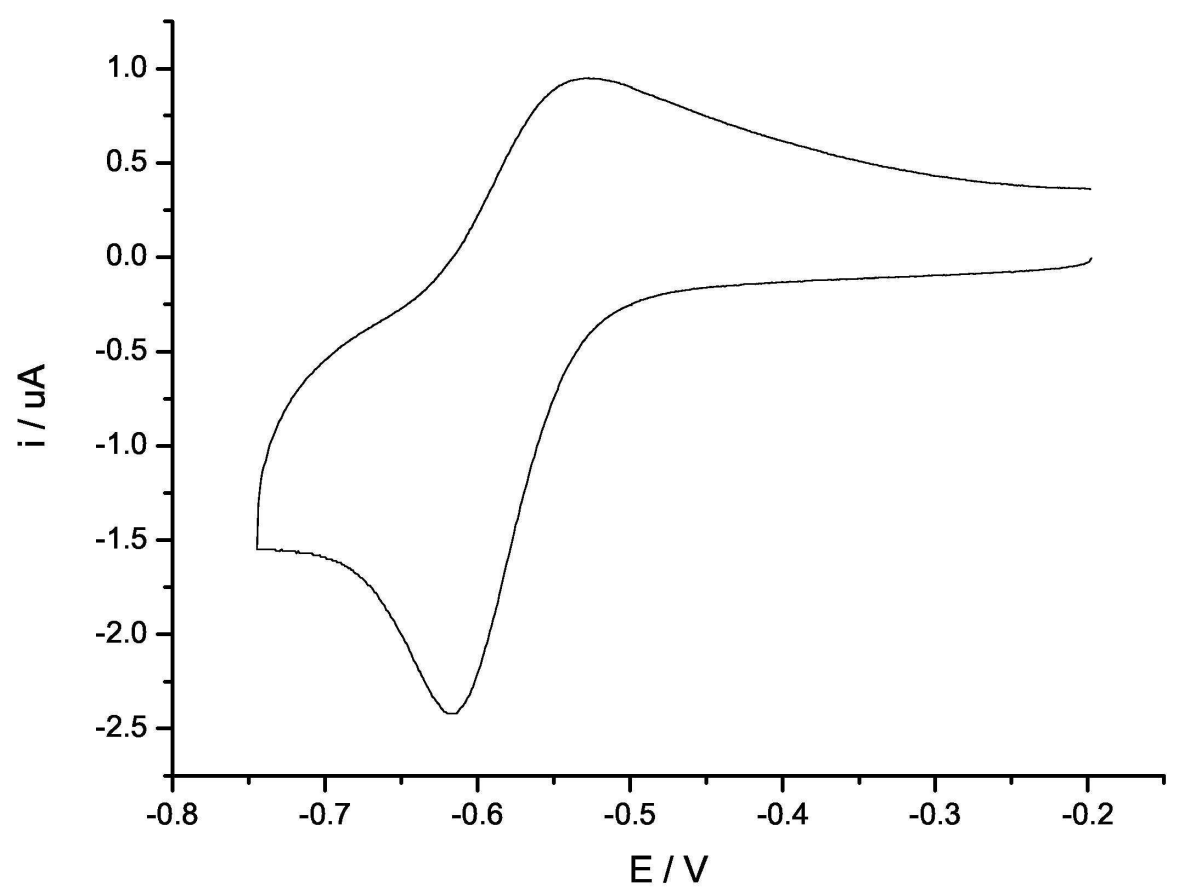

$100 \times 79 \mathrm{~mm}(600 \times 600 \mathrm{DPI})$ 
1

2

3

4

5

6

7

8

9

10

11

12

13

14

15

16

17

18

19

20

21

22

23

24

25

26

27

28

29

30

31

32

33

34

35

36

37

38

39

40

41

42

43

44

45

46

47

48

49

50

51

52

53

54

55

56

57

58

59

60

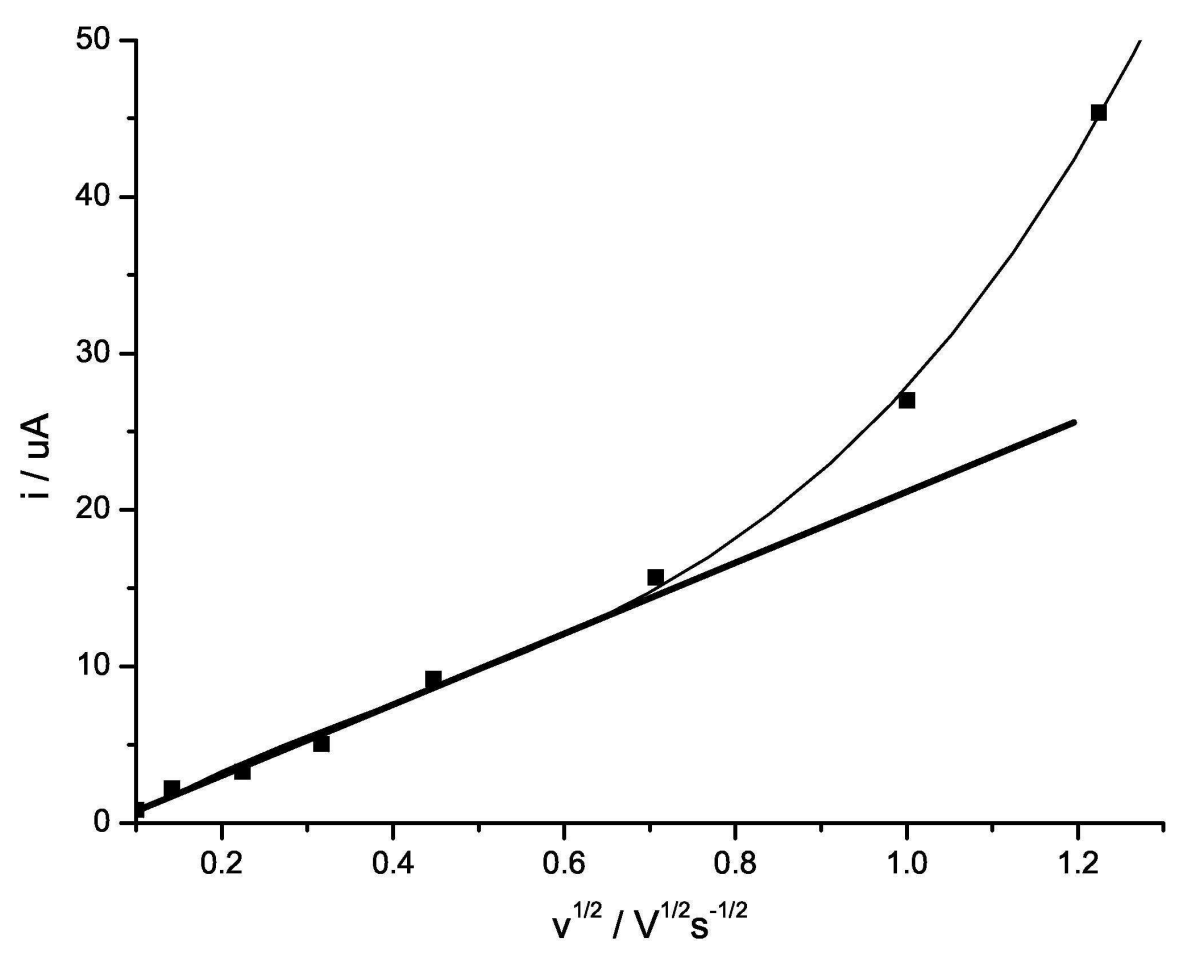

Figure 6. The dependence of cathodic peak current on scan rate for AQ- $\beta-C D$ solution. Supporting electrolyte: $0.1 \mathrm{M}$ phosphate buffer $+25 \% \mathrm{DMF}, \mathrm{pH} 8.9$.

$97 \times 81 \mathrm{~mm}(600 \times 600 \mathrm{DPI})$ 
Figure 7. Cyclic voltammograms recorded using (a) gold electrode and electrode modified by: (b) mixture of $\mathrm{N}$-(1-adamantane) lipoamide and hexanethiol, (c) hexanethiol, in phosphate buffer containing $25 \%$ DMF, pH 8.9. All electrodes were kept in $0.1 \mathrm{mM}$ solution of mono-6-deoxy-6thioureido-(1-anthraquinone)-per-0-methyl- $\beta$-cyclodextrin for two hours. $100 \times 79 \mathrm{~mm}(600 \times 600$ DPI $)$ 